\title{
[JPDATE Abnormal uterine bleeding
}

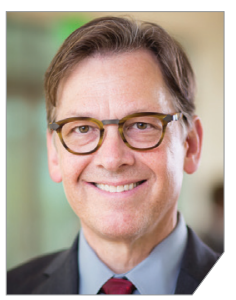

Howard T. Sharp, MD

Dr. Sharp is Professor and Vice Chair

for Clinical Activities, Department of Obstetrics and Gynecology, University of Utah Health, Salt Lake City.

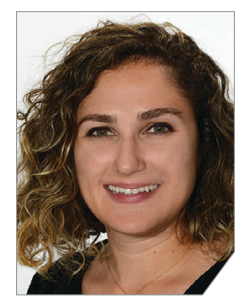

Evangelia Lea Lazaris, MD

Dr. Lazaris is a Resident in the Department of Obstetrics and Gynecology, University of Utah Health.

The evidence base on management strategies for AUB continues to grow, and recent studies suggest that therapy can be tailored based on certain factors, such as patient age and fertility goals and surgeon skill

\section{IN THIS ARTICLE}

Cyclic progestogens page 36

\section{Endometrial ablation}

page 37

\section{Isthmocele} management page 38
A bnormal uterine bleeding (AUB) continues to be a top reason that women present for gynecologic care. In general, our approach to the management of AUB is to diagnose causes before we prescribe therapy and to offer conservative therapies initially and progress to more invasive measures if indicated.

In this Update, we highlight several new studies that provide evidence for preferential use of certain medical and surgical therapies. In considering conservative therapy for the treatment of AUB, we take a closer look at the efficacy of cyclic progestogens. Another important issue, as more types of endometrial ablation (EA) are being developed and are coming into the market, is the need for additional guidance regarding decisions about EA versus progestin-releasing intrauterine devices (IUDs). Lastly, an unintended consequence of an increased cesarean delivery rate is the development of isthmocele, also known as cesarean scar defect or uterine niche. These defects, which can be bothersome and cause abnormal bleeding, are treated with various techniques. Within the last year, 2 systematic reviews that compare the efficacy of several different approaches and provide guidance have been published.

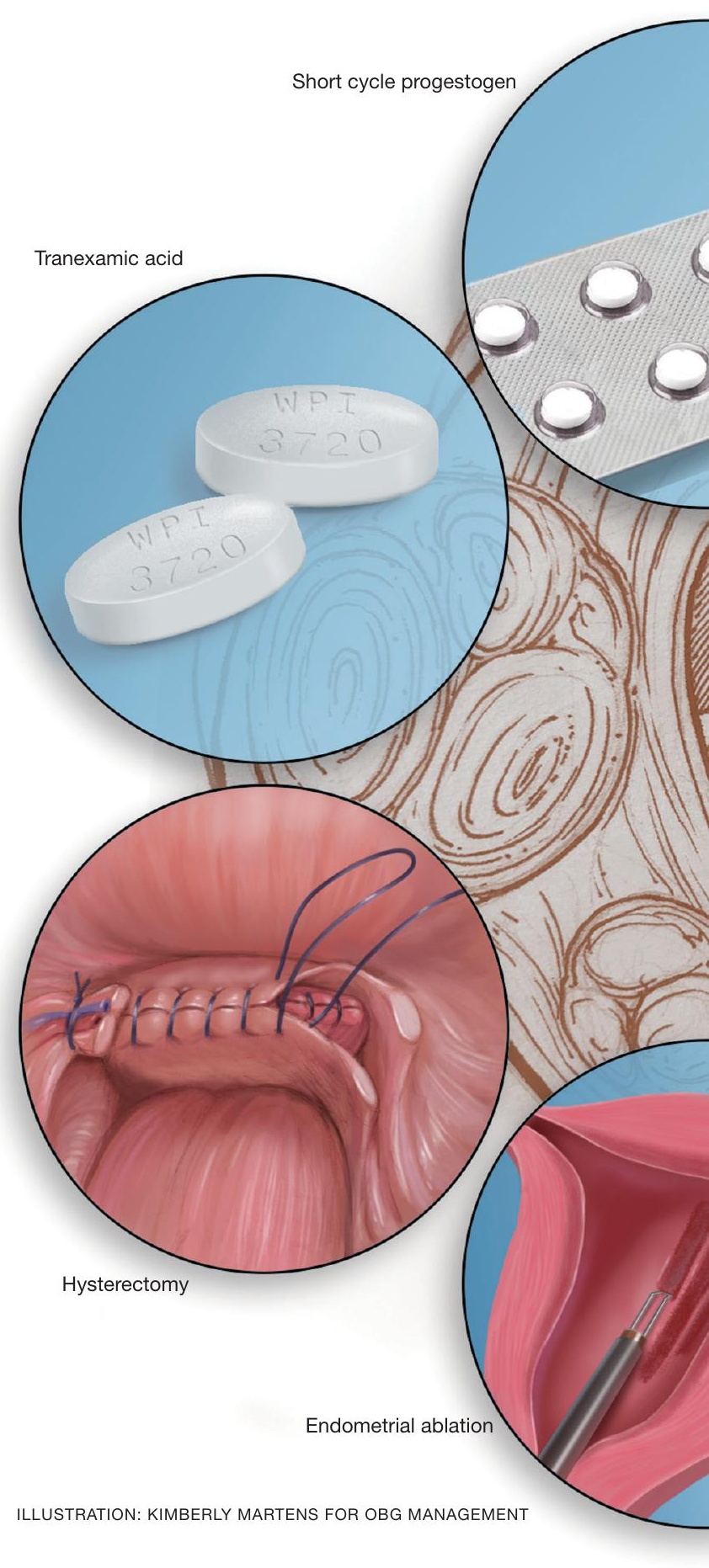




\section{Is it time to retire cyclic progestogens for the treatment of heavy menstrual bleeding?}

Bofill Rodriguez M, Lethaby A, Low C, et al. Cyclical progestogens for heavy menstrual bleeding. Cochrane Database Syst Rev. 2019;(8):CD001016.

$\mathrm{n}$ a recent Cochrane Database Systematic Review, Bofill Rodriguez and colleagues looked at the efficacy, safety, and tolerability of oral progestogen therapy for heavy menstrual bleeding. ${ }^{1}$ They considered progestogen (medroxyprogesterone acetate or norethisterone) in short-cycle use (7 to 10 days in the luteal phase) and long-

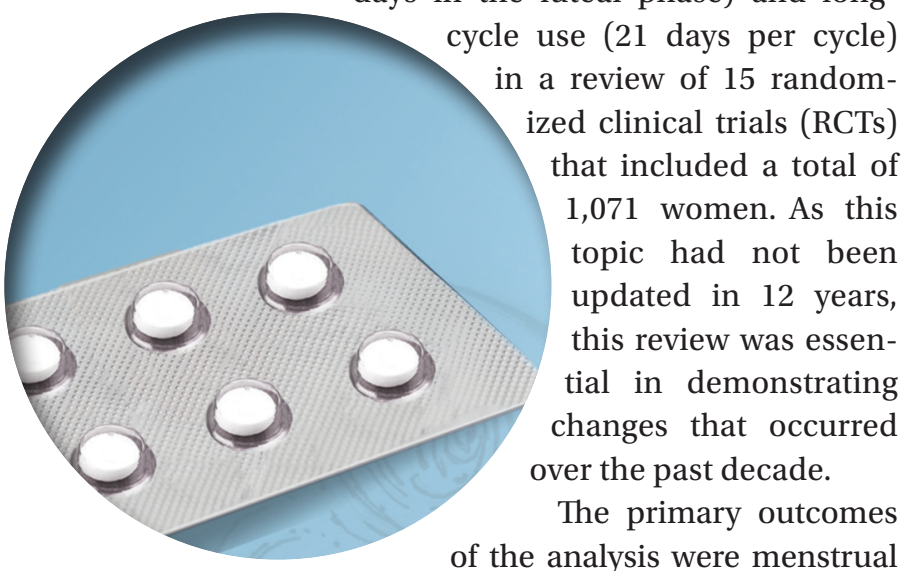

blood loss and treatment satisfaction. Secondary outcomes included the number of days of bleeding, quality of life, adherence and acceptability of treatment, adverse events, and costs.

\section{Classic progestogens fall short compared with newer approaches}

Analysis of the data revealed that shortcycle progestogen was inferior to treatment with tranexamic acid, danazol, and the 65- $\mu$ g progesterone-releasing IUD (Pg-IUD). Of note, the 65- $\mu$ g Pg-IUD has been off the market since 2001, and danazol is rarely used in current practice.
Furthermore, based on 2 trials, cyclic progestogens demonstrated no clear benefit over nonsteroidal anti-inflammatory drugs. Additionally, long-cycle progestogen therapy was found to be inferior to the 52-mg levonorgestrel-releasing IUD (LNG-IUD), tranexamic acid, and ormeloxifene.

It should be noted that the quality of evidence is still lacking for progestogen therapy, and this study's main limitation is bias, as the women and the researchers were aware of the treatments that were given. This review is helpful, however, for emphasizing the advantage of tranexamic acid and LNG-IUD use in clinical care.

The takeaway. Although it may not necessarily be time to retire the use of cyclic oral progestogens, the 52-mg LNG-IUD or tranexamic acid may be more successful for treating AUB in women who are appropriate candidates.

\section{WHAT THIS EVIDENCE MEANS FOR PRACTICE}

Cyclic progestogen therapy appears to be less effective for the treatment of AUB when compared with tranexamic acid and the LNG-IUD. It does not appear to be more helpful than nonsteroidal antiinflammatory drugs. We frequently offer and prescribe tranexamic acid, 1,300 mg 3 times daily, as a medical alternative to hormonal therapy for up to 5 days monthly for women without thromboembolism risk. Lukes and colleagues published an RCT in 2010 that demonstrated a 40\% reduction of bleeding in tranexamic acid-treated women compared with an $8.2 \%$ reduction in the placebo group. ${ }^{2}$ 


\section{Endometrial ablation: New evidence informs when it could (and could not) be the best option}

\begin{abstract}
Bergeron C, Laberge PY, Boutin A, et al. Endometrial ablation or resection versus levonorgestrel intra-uterine system for the treatment of women with heavy menstrual bleeding and a normal uterine cavity: a systematic review with meta-analysis. Hum Reprod Update. 2020;26:302-311.
\end{abstract}

Vitale SG, Ferrero S, Ciebiera M, et al. Hysteroscopic endometrial resection vs hysterectomy for abnormal uterine bleeding: impact on quality of life and sexuality. Evidence from a systematic review of randomized controlled trials. Curr Opin Obstet Gynecol. 2020;32:159-165.

T wo systematic reviews evaluated the efficacy of EA in women with abnormal uterine bleeding. One compared EA with the LNG-IUD and reported on safety and efficacy, while the other compared EA with hysterectomy and reported on quality of life.

Bergeron and colleagues reviewed 13 studies that included 884 women to compare the efficacy and safety of EA or resection with the LNG-IUD for the treatment of premenopausal women with AUB. ${ }^{3}$ They found no significant differences between EA and the LNG-IUD in terms of subsequent hysterectomy (risk ratio $[\mathrm{RR}]=1.3$; 95\% confidence interval $[\mathrm{CI}]$, $0.60-2.11)$. It was not surprising that, when looking at age, EA was associated with a higher risk for hysterectomy in women younger than age $42(\mathrm{RR}=5.26$; 95\% CI, 1.21-22.91). Conversely, subsequent hysterectomy was less likely with EA compared to LNG-IUD use in women older than 42 years. However, statistical significance was not reached in the older group $(\mathrm{RR}=$ 0.51 ; 95\% CI, 0.21-1.24).
In the systematic review by Vitale and colleagues, 9 studies met inclusion criteria for a comparison of EA and hysterectomy, with the objective of ascertaining improvement in quality of life and several other measures. ${ }^{4}$

Although there was significant heterogeneity between assessment tools, both treatment groups experienced similar improvements in quality of life during the first year. However, hysterectomy was more advantageous in terms of improving uterine bleeding and satisfaction in the long term when compared with EA. ${ }^{4}$

The takeaway. The LNG-IUD continues to be a very good option to treat AUB in patients who would be candidates for EA, especially in younger patients, who have a high failure rate with EA. Hysterectomy may have greater durability for improving quality of life and bleeding compared with EA.

\section{WHAT THIS EVIDENCE MEANS FOR PRACTICE}

As EA is considered, it is important to continue to counsel about the efficacy of the LNG-IUD, as well as its decreased associated morbidity. Additionally, EA is particularly less effective in younger women. 


\section{Laparoscopy is best approach for isthomocele management, with caveats}

He Y, Zhong J, Zhou W, et al. Four surgical strategies for the treatment of cesarean scar defect: a systematic review and network meta-analysis. J Minim Invasive Gynecol. 2020;27:593-602.

Vitale SG, Ludwin A, Vilos GA, et al. From hysteroscopy to laparoendoscopic surgery: what is the best surgical approach for symptomatic isthmocele? A systematic review and meta-analysis. Arch Gynecol Obstet. 2020;301:33-52.

T he isthmocele (cesarean scar defect, uterine niche), a known complication of cesarean delivery, represents a myo-

$\overline{\text { FAST }}$

TRACK

In terms of reducing abnormal bleeding and scar depth, laparoscopy for isthmocele performed better than hysteroscopy, laparoscopy combined with hysteroscopy, and vaginal repair metrial defect in the anterior uterine wall that often presents as abnormal uterine bleeding. It also can be a site for pregnancy-related complications, such as invasive placentation, placenta previa, and uterine rupture.

Two systematic reviews compared surgical strategies for treating isthmocele, including laparoscopy, hysteroscopy, combined laparoscopy and hysteroscopy, laparotomy, and vaginal repair.

\section{Laparoscopy reduced isthmocele-associated AUB better than other techniques}

A review by $\mathrm{He}$ and colleagues analyzed data from 10 pertinent studies (4 RCTs and 6 observational studies) that included 858 patients in total. ${ }^{5}$ Treatments compared were laparoscopy, hysteroscopy, combined laparoscopy with hysteroscopy, and vaginal repair for reduction of AUB and isthmocele and diverticulum depth.

The authors found no difference in intraoperative bleeding between the 4 surgical methods (laparotomy was not included in this review). Hysteroscopic surgery was associated with the shortest operative time, while laparoscopy was the longest surgery. In terms of reducing intermittent abnormal bleeding and scar depth, laparoscopic surgery performed better than the other 3 methods.

\section{Approach considerations in isthmocele repair}

Vitale and colleagues conducted a systematic review that included 33 publications (28 focused on a single surgical technique, 5 compared different techniques) to examine the effectiveness and risks of various surgical approaches for isthmocele in women with AUB, infertility, or for prevention of obstetric complications. ${ }^{6}$

Results of their analysis in general favored a laparoscopic approach for patients who desired future fertility, with an improvement rate of $92.7 \%$. Hysteroscopic correction had an $85 \%$ improvement rate, and vaginal correction had an $82.5 \%$ improvement rate.

Although there were no high-level data to suggest a threshold for myometrial thickness in recommending a surgical approach, the authors provided a helpful algorithm for choosing a route based on a patient's fertility desires. For the asymptomatic patient, they suggest no treatment. In symptomatic patients, the laparoscopic approach is the gold standard but requires significant laparoscopic surgical skill, and a hysteroscopic approach may be considered as an alternative route if the residual myometrial defect is greater than 2.5 to $3.5 \mathrm{~mm}$. For patients who are not considering future reproduction, hysteroscopy is the gold standard as long as the residual myometrial thickness is greater than 2.5 to $3.5 \mathrm{~mm}$.

The takeaway. Of the several methods used for surgical isthmocele management, the laparoscopic approach reduced 


\section{UPDATE abnormal uterine bleeding}

CONTINUED FROM PAGE 38

intermittent abnormal bleeding and scar depth better than other methods. It also was associated with the longest surgical duration. Hysteroscopic surgery was the quickest procedure to perform and is effective in removing the upper valve to promote the elimination of the hematocele and symptoms of abnormal bleeding; however, it does not change the anatomic aspects of the isthmocele in terms of myometrial thickness. Some authors suggested that deciding

\section{WHAT THIS EVIDENCE MEANS FOR PRACTICE}

In terms of isthmocele repair, the laparoscopic approach is preferred in patients who desire fertility, as long as the surgeon possesses the skill set to perform this difficult surgery, and as long as the residual myometrium is thicker than 2.5 to $3.5 \mathrm{~mm}$.

on the surgical route should be based on fertility desires and the residual thickness of the myometrium.

\section{References}

1. Bofill Rodriguez M, Lethaby A, Low C, et al. Cyclical progestogens for heavy menstrual bleeding. Cochrane Database Syst Rev. 2019;(8):CD001016.

2. Lukes AS, Moore KA, Muse KN, et al. Tranexamic acid treatment for heavy menstrual bleeding: a randomized controlled study. Obstet Gynecol. 2010;116:865-875.

3. Bergeron C, Laberge PY, Boutin A, et al. Endometrial ablation or resection versus levonorgestrel intra-uterine system for the treatment of women with heavy menstrual bleeding and a normal uterine cavity: a systematic review with metaanalysis. Hum Reprod Update. 2020;26:302-311.

4. Vitale SG, Ferrero S, Ciebiera M, et al. Hysteroscopic endometrial resection vs hysterectomy for abnormal uterine bleeding: impact on quality of life and sexuality. Evidence from a systematic review of randomized controlled trials. Curr Opin Obstet Gynecol. 2020;32:159-165.

5. He Y, Zhong J, Zhou W, et al. Four surgical strategies for the treatment of cesarean scar defect: a systematic review and network meta-analysis. J Minim Invasive Gynecol. 2020;27:593-602.

6. Vitale SG, Ludwin A, Vilos GA, et al. From hysteroscopy to laparoendoscopic surgery: what is the best surgical approach for symptomatic isthmocele? A systematic review and metaanalysis. Arch Gynecol Obstet. 2020;301:33-52. 